\title{
15 Sexuell übertragbare Infektionen
}

\author{
Falk R. Ochsendorf und Helmut Schöfer
}

\subsection{Grundlegende Überlegungen}

\subsubsection{Grundmuster „Sexuell übertragbarer Infektionen“ (STI)}

Sexuell übertragbare Erkrankungen sind genitale/anale/orale Kontaktinfektionen.

Sexuell übertragbare Infektionen wurden früher als „Geschlechtskrankheiten“ bezeichnet. Man bezeichnet damit genitale Kontaktinfektionen, die in der Regel durch Geschlechtsverkehr übertragen werden und Symptome vorwiegend in der Genitalregion hervorrufen. Sie werden durch eine Vielfalt von Erregern (Bakterien, Viren, Pilze, Parasiten) hervorgerufen (s. Tab. 1). Die WHO schätzt, dass weltweit täglich eine Million Neuinfektionen auftreten (CDC 2006). Seit dem Jahr 2001 besteht in Deutschland keine Meldepflicht mehr für die klassischen Geschlechtskrankheiten, nur eine Labormeldepflicht für HIV-Infektion und Syphilis.

Menschen mit erhöhtem Risiko für STI zeigen häufige Partnerwechsel. Zudem entscheidet der Zugang zu medizinischer Versorgung, d.h. frühzeitiger Unterbrechung von Infektionsketten, über die epidemiologische Situation. Am häufigsten findet man STI's im frühen Erwachsenenalter, bei Männern doppelt so häufig wie bei Frauen. Die Inzidenz in Großstädten ist am höchsten, auf dem Land am geringsten. Bestimmte Berufe, wie z.B. Sexworker (Prostitutierte u.a.), haben ein höheres Infektionsrisiko, daneben aber auch Personen unter zeitweiliger erzwungener sexueller Enthaltsamkeit, wie Soldaten und Schiffsbesatzungen. 
Tab. 1 Übersicht über Erreger, Erkrankung und Erkrankungsmuster der sexuell übertragbaren Infektionen (C. = Chlamydia trachomatis, LGTI = lower genital tract infection, UGTI = upper genital tract infection, $\mathrm{N}=$ Neisseria, NGU = nicht gonorrhoische Urethritis, spp. = Spezies)

\begin{tabular}{|c|c|c|c|c|c|c|c|c|c|}
\hline \multirow[b]{2}{*}{ Erreger } & \multirow{2}{*}{\multicolumn{2}{|c|}{ Erkrankung }} & \multicolumn{7}{|c|}{ Erkrankungsmuster } \\
\hline & & & $\stackrel{气}{\Xi}$ & $\bar{E}$ & E & 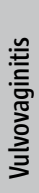 & 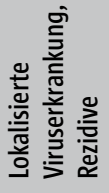 & 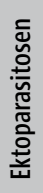 & 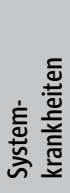 \\
\hline \multirow{9}{*}{ Bakterien } & Treponema pallidum & Syphilis & + & & & & - & & + \\
\hline & N. gonorrhoeae & Gonorrhoe & & + & + & & & & \\
\hline & Hämophilus ducrey & Ulcus molle & + & & & & & & \\
\hline & Klebsiella granulomatis & $\begin{array}{l}\text { Granuloma } \\
\text { inguinale }\end{array}$ & + & & & & & & \\
\hline & C. trachomatis L1-3 & $\begin{array}{l}\text { Lymphogranuloma } \\
\text { venereum }\end{array}$ & + & & & & & & \\
\hline & C. trachomatis D-K & NGU/Adnexitis & & + & + & & & & \\
\hline & $\begin{array}{l}\text { Mycoplasma spp. } \\
\text { (hominis, genitalium) }\end{array}$ & $\mathrm{NGU}$ & & + & & & & & \\
\hline & Ureapasma urealyticum & NGU & & + & & & & & \\
\hline & Gardnerella vaginalis & & & & & + & & & \\
\hline \multirow{6}{*}{ Viren } & Herpes simplex Virus 1,2 & Herpes genitalis & & & & & + & & \\
\hline & Cytomegalievirus & Ulzera bei HIV & + & & & & & & \\
\hline & HPV & $\begin{array}{l}\text { Condylomata } \\
\text { acuminata, } \\
\text { bowenoide } \\
\text { Papulose }\end{array}$ & & & & & + & & \\
\hline & Molluscum contagiosum & Dellwarzen & & & & & + & & \\
\hline & Hepatitis B, C & Hepatitis & & & & & & & + \\
\hline & HIV & AIDS & & & & & & & + \\
\hline Pilze & Candida spp. & $\begin{array}{l}\text { Balanoposthitis, } \\
\text { Vulvovaginitis }\end{array}$ & & & & & & & \\
\hline Protozoen & Trichomonas vaginalis & $\begin{array}{l}\text { Vulvovaginitis, } \\
\text { Urethritis }\end{array}$ & & + & & + & & & \\
\hline \multirow{2}{*}{ Parasiten } & Phtirus pubis & Pediculosis pubis & & & & & & + & \\
\hline & Sarcoptes scabiei & Skabies & & & & & & + & \\
\hline
\end{tabular}

Verschiedene Erreger verursachen gleichartige Erkrankungsmuster. 
Dennoch findet man bei verschiedenen Erregern gemeinsame pathologische Grundmuster:

- Manifestation mit einem Ulcus an der Eintrittspforte und lymphogener Ausbreitung,

- Entzündungen mit Ausfluss als Folge nicht aszendierender Infektionen des unteren Genitaltrakts (= „lower genital tract infection, LGTI“)

- Aufsteigen dieser Infektionen in die Hohlorgane des Urogenitaltrakts (= „upper genital tract infection“, UGTI)

- Vulvovaginitis

- lokalisierte Viruserkrankungen mit Neigung zu Rezidiven

- Ektoparasitosen

- Systemkrankheiten

\subsubsection{Gemeinsame Eigenschaften der STI-Erreger}

Neben den gleichen Symptom-Mustern verbindet diese Erreger ihre Spezialisierung auf den Menschen sowie die Genitalgegend. Die Erreger sind gegenüber Umwelteinflüssen, wie Austrocknen, sehr empfindlich. Dies bedingt eine kurze Überlebenszeit außerhalb des Körpers, eine indirekte Übertragung ist nur in seltenen Fällen möglich. In der Regel ist ihre Infektiosität gering, sodass längere physikalische Kontakte zur Weitergabe der Infektion nötig sind. Diese Eigenschaften erklären, dass die Erreger v.a. beim Geschlechtsverkehr von Mensch zu Mensch übertragen werden. Wegen dieses gemeinsamen Übertragungswegs werden oft mehrere STI-Erreger gleichzeitig übertragen. Je nach Inkubationszeit verursachen die verschiedenen Organismen dann Symptome zu unterschiedlichen Zeitpunkten. Das Vorhandensein einer STI-Erkrankung zeigt an, dass Geschlechtsverkehr mit einem mit unbekanntem Erreger-Spektrum infizierten Menschen stattgefunden hat. Sie muss daher den Arzt veranlassen, nach weiteren STI-Erregern zu suchen.

\subsubsection{Schwierigkeiten bei der Bekämpfung}

Die Diagnose einer STI bedeutet: Partnerdiagnostik und -therapie.

Die Erreger verursachen, zumindest vorübergehend, geringe oder keine Beschwerden, können aber weitergegeben werden. Damit entziehen sie sich einer Diagnostik oder Therapie. Nach erfolgreicher Behandlung besteht keine Immunität, was auch die Entwicklung von Impfungen bisher verhindert hat (Ausnahme: Hepatitis B). Virale Erkrankungen lassen sich nicht komplett beseitigen, da es sich um latente chronische Infektionen handelt. Dies erklärt die Rezidivneigung. Die Verknüpfung mit dem Geschlechtstrieb führt dazu, dass rationale Aufklärung nur begrenzt wirkt. Die Taburegion „Genitalbereich“ sowie damit verbundene psychologische Faktoren hindern an der Aufdeckung und Unterbrechung von Infektionsketten. Hierzu ist die Untersuchung und Behandlung der Geschlechtspartner, d.h. derjenigen vor Beginn der Symptome und nach Auftreten, entsprechend der Inkubationszeiten der Erreger essenziell. Die Aufdeckung dieser Kontakte führt beim Patienten zu Konfliktsitua- 
tionen in der Partnerschaft, teils auch zu Beeinträchtigungen sozialer Kontakte. Daher wird von manchen Erkrankten die Möglichkeit einer sexuell übertragbaren Erkrankung völlig abgelehnt, bzw. die Preisgabe (v.a. außerehelicher) Sexualpartner vermieden.

\title{
15.2 Leitsymptom: Ulkus
}

\author{
Ulzera und entzündliche Genitalerkrankungen erhöhen das Übertragungs- \\ risiko für HIV.
}

\subsubsection{Syphilis}

Die Inzidenz der Syphilis nimmt vor allem im Kollektiv der „Männer die Sex mit Männern haben (MSM)“ wieder zu (Schöfer 2010). Die erste Inkubationszeit beträgt 3 Wochen, dann tritt ein schmerzloses Ulkus mit einem harten Rand an der Stelle des Erregereintritts in die Haut sowie eine symptomlose Schwellung der regionären Lymphknotenstationen auf. Die Diagnostik erfolgt in diesem Stadium über Dunkelfeldmikroskopie oder die Serologie (TPPA, TPHA, FTA-ABS u.a. spezifische Reaktionen) (Lautenschlager 2006). Unbehandelt können in der Folge eine generalisierte Lymphknotenschwellung, Syphilide (= vielgestaltige Exantheme), luetische Papeln (= erregerreiche Papeln wie Condylomata lata oder Plaques muceuses), spezifischer „mottenfraßähnlicher“ Haarausfall durch Erregervermehrung in der Kopfhaut und das syphilitische Leukoderm am Hals auftreten. Diese Symptome sind Zeichen einer Bakteriämie durch T. pallidum. Im Rahmen der HIV-Infektion findet man teils atypische Verläufe.

Die Therapie erfolgt gemäß den aktuellen Leitlinien mit Penicillin (DSTDG 2010).

\subsubsection{Ulcus molle}

Die Erkrankung ist in Westeuropa selten. Endemisch ist die Erkrankung in Südostasien und Afrika. Die Inkubationszeit beträgt 1-5 Tage, in Ausnahmefällen bis zu 30 Tagen. Es entstehen meist multiple, sehr schmerzhafte, scharf begrenzte Ulzera mit ausgefransten, überhängenden, weichen Rändern. Typisch ist der brennende Schmerz beim Kontakt mit Urin. Die regionären Lymphknoten sind meist einseitig schmerzhaft vergrößert. Der Erregernachweis ist schwierig. Die Therapie erfolgt einmalig mit mindestens $250 \mathrm{mg}$ Ceftriaxon i.m. oder Azithromycin $1 \mathrm{~g}$ oral (Lewis 2003).

\subsubsection{Lymphogranuloma venereum}

Die Erkrankung ist endemisch in Entwicklungs- und tropischen Ländern. Nach einer Inkubationszeit von 7-10 (3-30) Tagen tritt ein Herpes-simplex-ähnliches Bläschen auf, das ulzeriert und nach ca. 14 Tagen abheilt. Etwa 3-4 Wochen nach Infektion tritt das Sekundärstadium mit Krankheitsgefühl und Temperaturanstieg auf verbunden mit schmerzhaften, einseitigen Schwellungen der Leistenlymphknoten (bis 
faustgroß, gerötet, mit der Unterlage und der darüberliegenden Haut verbacken). Gefürchtet sind die Komplikationen durch Einschmelzung und Ausbreitung in Form destruierender granulomatöser Entzündungen um die äußeren Geschlechtsorgane, Analgegend und Rektum mit konsekutiver Fistelbildung. Der Erregernachweis erfolgt mit PCR/LCR aus Zervix, Urethra und/oder Rektum, die Therapie mit Doxycylin 2 x $100 \mathrm{mg} / \mathrm{d}$ über 3 Wochen (White 2009).

\subsubsection{Granuloma inguinale}

Auch diese Erkrankung findet man vor allem in tropischen und subtropischen Gebieten. Die Inkubationszeit wird auf 8-80 Tage geschätzt. Dann treten schmerzlose, derbe entzündliche subkutane Papeln und Knoten auf, die sich in Geschwüre und langsam zunehmende granulomatöse Massen umwandeln. Durch Ausbreitung können so ausgedehnte schmerzlose, verkrustete ulzero-granulomatöse Läsionen entstehen, die in der Folge fibrosieren und zu Strikturen führen. Die Diagnose wird durch Biopsie und Gewebsausstriche gestellt. Die Therapie erfolgt mit Tetrazyklinen (Doxycylin 2 x $100 \mathrm{mg} / \mathrm{d}$ ) oder Cotrimoxazol (2 Tabl. à 160/80o mg/d) über 3 Wochen (O’Farrell 2002).

\subsubsection{Herpes simplex Infektion}

Unterscheiden muss man die Erstinfektion (Balanoposthitis herpetica) und den rezidivierenden Herpes genitalis. Der Erstkontakt führt in den meisten Fällen zu subklinischen Infektionen. Lediglich bei etwa 1\% kommt es 3-10 Tage nach dem Kontakt zum Auftreten zahlreicher schmerzhafter gruppierter Bläschen mit Rötung und Schwellung, hohem Fieber, und beidseitiger Lymphadenitis. Die Veränderungen heilen nach etwa 3 Wochen ab. Bei Reaktivierung sind die Veränderungen meist geringer bezüglich Ausdehnung, Entzündung und Schmerzhaftigkeit. Es fehlen Systemzeichen. Die Hautveränderungen heilen nach 5-8 Tagen ab. Therapie der Wahl ist Aciclovir oral, ggf. auch als Dauerprophylaxe.

\subsubsection{Cytomegalievirus-Infektion}

Hautmanifestationen können bei immunsupprimierten Patienten (Organtransplantation, HIV-Infektion) auftreten. An der Haut finden sich dann chronische, sehr schmerzhafte Geschwüre im Ano-Perinealbereich, ähnlich einem vegetierenden Herpes simplex genitoanalis. Die Therapie erfolgt mit Ganciclovir oder Foscarnet.

\subsection{Leitsymptom: Ausfluss}

\subsubsection{Gonorrhoe}

Gonokokken haben eine Prädilektion für Zylinderepithel. Nach einer Inkubationszeit von 2-4 Tagen (1-10 Tage) entstehen Entzündungen an Urethra, nach Aszension auch Prostata und Nebenhoden, bei der Frau an Urethra, Zervix und Tuben, bei beiden Geschlechtern evtl. auch in Rektum und Pharynx. Die akute Gonorrhoe beim Mann 
zeigt sich durch Brennen in der Harnröhre, v.a. beim Urinieren, und Auftreten eines eitrigen, „rahmigen“ gelb bis gelb-grünlichen Fluor urethralis. Bei fehlender Behandlung und Aszension des Erregers können weitere Symptome hinzutreten. Der Nachweis erfolgt mittels direktem Erregernachweis und Kultur. Zur Therapie werden Cephalosporine der dritten Generation wie Cefixim (einmalige orale Gabe von $400 \mathrm{mg}$ p.o.) oder Ceftriaxon (einmalige Gabe von 125-250 mg i.m.) empfohlen (Kohl 2009).

\subsubsection{Nicht-gonorrhoische Urethritis (NGU)}

Hat ein Patient Beschwerden wie bei einer Gonorrhoe, ohne dass sich Gonokokken nachweisen lassen, spricht man von einer NGU. Die relevantesten Erreger hierfür sind Chlamydien, Ureaplasmen und Trichomonaden (Yokoi et al. 2007). Die Inkubationszeit beträgt 4 Tage (Trichomonaden) bis 1-3 Wochen (Mykoplasmen). Zur Diagnostik färbt man einen Urethralabstrich. Finden sich > 4 Leukozyten pro Gesichtsfeld liegt eine Urethritis vor. Zum Erregernachweis werden molekularbiologische Methoden (PCR, LCR) im Erststrahlurin verwendet bzw. im Urethralabstrich (Chlamydien). Ureaplasmen/Mykoplasmen können daraus angezüchtet werden. Trichomonaden-Infektionen beim Mann sind meist asymptomatisch. Zum Nachweis verwendet man zentrifugierten Morgenurin oder eine Kultur. Die Behandlung erfolgt mit Doxycylin, Makroliden oder Chinolonen über 1 (Chlamydien) bis 3 Wochen (Mykoplasmen). Trichomonaden werden mit Metronidazol behandelt (einmalig $2 \mathrm{~g}$ ).

Gefürchtete Komplikation einer Chlamydieninfektion ist die Übertragung auf die weibliche Partnerin. Dies kann zur Adnexitis mit nachfolgender Sterilität führen (Clad u. Krause 2007).

Papeln im Genitalbereich erfordern eine Syphilis- und HIV-Serologie.

\subsection{Leitsymptom: Papeln}

Hautfarbene meist symptomlose Papeln können durch Humane Papillomviren (HPV) hervorgerufen werden. Meist handelt es sich um sogenannte „low-risk“ Typen (HPV $6,11)$. Die klinische Präsentation reicht von flachen unscheinbaren Papeln über hautfarbene bis rötliche weiche papillomatöse Knoten zu beetartigen Riesenkondylomen. Abzugrenzen sind diese von den „Condylomata lata“ der Syphilis. Mollusken, glasige, glänzende, zentral eingesunkene Papeln im Genitalbereich bei Erwachsenen sollten Anlass sein, nach einer HIV-Infektion zu fanden.

Unerträglicher (,,bestialischer") Juckreiz, v.a. nachts, ist verdächtig für eine Skabies-Infektion. 


\subsection{Leitsymptom: Juckreiz}

Juckreiz am Penis und unter der Vorhaut, oft verbunden mit einer Rötung und weißen Belägen, sind Hinweis auf eine Candida-Infektion (Behandlung: z.B. Nystatin-Paste). Juckende Ekzeme im Genitalbereich sollten an einen Befall durch Filzläuse denken lassen. Typisch sind kleine bläuliche Flecke in der Genital-, Unterbauch und Inguinalregion als Ausdruck der Bissstellen der Läuse (Behandlung: Permethrin topisch).

Ein generalisiertes, quälend juckendes, von Kratzeffekten dominiertes, papulo-vesikulo-squamokrustöses Exanthem (,ekzemartig“) muss an eine Skabies denken lassen. Prädilektionsstellen sind die Genitalregion, Axillen, Bauchnabel, Mamillen, Fingerzwischenräume und Handgelenke. Bei genauer Inspektion lassen sich hier mehrere Millimeter lange, strichförmige Gänge finden. Die Behandlung erfolgt einmalig mit 5\% Permethrin-Salbe topisch. Wichtig ist die gleichzeitige Behandlung aller (auch beschwerdefreier!) Kontaktpersonen (Hicks u. Elston 2009).

\subsection{Leitsymptom: Exanthem}

Exantheme können viele Ursachen haben. Neben Arzneimitteln ist immer auch an eine Syphilis als Auslöser zu denken. Insbesondere wenn das Exanthem symptomlos ist, sollten Handflächen und Fußsohlen inspiziert, alle Lymphknotenstationen abgetastet und eine Syphilis-Serologie veranlasst werden.

\section{Fazit}

Die Diagnose jeder einzelnen STI muss die Suche nach weiteren STI nach sich ziehen (Symptome? Erreger?). Chlamydien-, Hepatitis B- und HIV-Infektionen können lange Zeit asymptomatisch verlaufen. Sie lassen sich nur erkennen, wenn aufgrund einer STI-Diagnose ein Erregernachweis (direkt oder serologisch) veranlasst wird.

\section{Literatur}

Centers for Disease Control and Prevention (2006) Sexually transmitted diseases treatment guidelines. MMWR Recomm Rep. 55, 1-94

Clad A, Krause W (2007) Urogenitale Chlamydieninfektionen bei Frau und Mann. Hautarzt 58, 13-17

Deutsche STD-Gesellschaft DSTDG (Hrsg.) (2010): Diagnostik und Therapie der Syphilis. http://www.awmf.org/ leitlinien/detail/ll/059-002.html, abgerufen am 20.10.2011

Hicks MI, Elston DM (2009) Scabies. Dermatol Ther 22, 279-292

Kohl PK (2009) Gonorrhoe. In: Schöfer H, Baur-Beger S (Hrsg.) Derma-Net-Online, BBS Wiesbaden. http://www. derma-net-online.de/buch/kapitel2_7/Kap_2_7_2.pdf, abgerufen am 04.06.2010

Lautenschlager S (2006) Syphilisdiagnostik: Klinische und labormedizinische Problematik. I Dtsch Dermatol Ges 4, 1058-1075

Lewis DA (2003) Chancroid: clinical manifestations, diagnosis, and management. Sex Transm Infect 79, 68-71

O'Farrell N (2002) Donovanosis. Sex Transm Infect 78, 452-457

Schöfer H (2010) Syphilis. In: Schöfer H, Baur-Beger S (Hrsg.) Derma-Net-Online 2010, BBS Wiesbaden. http:// www.derma-net-online.de/buch/kapitel2_7/Kap_2_7_1.pdf, abgerufen am 24.06.2010

White JA (2009) Manifestations and management of lymphogranuloma venereum. Curr Opin Infect Dis 22, 57-66

Yokoi S, Maeda S, Kubota Y et al (2007) The role of Mycoplasma genitalium and Ureaplasma urealyticum biovar 2 in postgonococcal urethritis. Clin Infect Dis 45, 866-871 